\title{
FPGA REALIZATION OF SIMPLIFIED LTE TURBO DECODER
}

\author{
E Sujatha1, C Subhas², M N Giri Prasad², N Padmaja ${ }^{3}$ \\ ${ }^{1}$ Research Fellow, Department of Electronics and Communications, Sree Vidyanikethan \\ Engineering College, Andhra Pradesh, India \\ ${ }^{2}$ Professor, Department of Electronics and Communications, JNTU College of Engineering, \\ Andhra Pradesh, India \\ ${ }^{3}$ Professor, Department of Electronics and Communications, Sree Vidyanikethan Engineering \\ College, Andhra Pradesh, India
}

\begin{abstract}
This article presents a simplified turbo decoder using Max-Log-Maximum a posteriori algorithm and its hardware realization. This work adopted Very large-scale Integration Optimization techniques of parallel computation, pipeline process for minimizing computation complexity and computation latency in each soft-input-softoutput decoder. Also, three-stage pipeline process of decoder was proposed here to improve functional performance and to reduce the hardware memory requirement. Further, performance re-timing, resource sharing and area optimization have been applied in implementation of the proposed turbo decoder. The proposed design is synthesized and implemented on Zynq FPGA board. The analysis and study of efficient resource utilization of the proposed design has been done and the proposed design achieved high throughput and area reduction by 40 to $50 \%$ than conventional designs.
\end{abstract}

Key words: Turbo codes, Max-Log-MAP algorithm, VLSI Design, Optimization

Cite this Article: E Sujatha, C Subhas, M N Giri Prasad, N Padmaja. FPGA Realization of Simplified LTE Turbo Decoder. International Journal of Electrical Engineering and Technology (IJEET). 12(9), 2021, pp. 121-131.

https://iaeme.com/Home/issue/IJEET? Volume $=12 \&$ Issue $=9$

\section{INTRODUCTION}

In recent wireless communication systems, reliable data transmission with high performance hardware realization is an ever-challenging task to a design engineer. Turbo code, a highperformance forward error correction (FEC) code, had been adopted as channel code [1-2] to modern wireless communication standards like $3^{\text {rd }}$ generation partnership project (3GPP) LTEAdvanced [3], 802.16m [1] and future broadcasting standard [4] due to their flexible and resource efficient utilization in its hardware realization. In the hardware realization of turbo decoder, decoding performance, power dissipation and hardware cost are the most important 
design considerations. To attain great decoding performance at the least expense of hardware implementation complexity in soft in soft out (SISO) decoder, many recent turbo decoder realizations applied the maximum a posteriori probability (MAP) algorithm [5] and its variants [6-9]. Here, some recent conventional designs of various novel architectural design aspects [23] and their performance analysis are specified below.

In 2011, VLSI architecture for modified Max-Log-MAP (MLMAP) algorithm with Mac Laurin Series had proposed by Rahul Shrestha in [13] to reduce the circuit complexity by replacing the multipliers with adders and shifters. Martina et al.[14] presented the state metric compression techniques, Non uniform quantization technique and Walsh - Hadamard transform to mitigate the power consumption of the decoder. Karim et al. [15] used Pipeline technique to reduce the critical path delay of the Add Compare Select Offset (ACSO) unit. In this design, interleaver memory was split into several small memory blocks and the row address was not changed during sub-block processing thus excessive switching in the router was reduced. Reference [16] detailed scalable MAP decoder designs which could support both single and double Binary CTC decoding and this design attain high area utilization. They presented parallel-window and hybrid-window MAP decoding. In [17], Yang Sun et al. proposed efficient hardware implementation of a highly-parallel 3GPP LTE/LTE-advance turbo decoder.

In 2013, Carlo Condo et al. [18] proposed a novel design of a reconfigurable architecture for both LDPC and turbo codes to solve the reconfiguration issue and proposed NoC-based turbo/LDPC decoder architecture with a small overhead. Rahul Shresta in [20] proposed a high throughput turbo decoder in $90 \mathrm{~nm}$ CMOS with 8 and 64 parallel radix-2 Map decoder architecture using the Log-MAP algorithm. This work proposed two schemes, a new ungrouped backward recursion scheme and a new state metric normalization technique to perform retiming and pipelining in architecture for performance improvement. Also, this work utilized fine grain clock gating technique to overcome power issue. In 2016, A. Li et al. in [21] proposed a fully parallel turbo decoding algorithm (FPTD), which permits parallel processing for higher throughput and minimizing computation complexity by 50\%. In 2019, Sujatha. E et al. presented optimized turbo decoder for performance improvement of turbo decoder, where parallel computation of state metrics optimized the hardware resource utilization, but, the present work is further improved design to optimize the turbo decoder functionality.

With these motivations, this novel work adopts the concept of 3 -stage pipelining, equivalent logic blocks and parallel process of loading, processing and pushing out the outputs used for decoding of multiple frames to minimize hardware requirement and improve the speed performance. In next Section, problem statement and design specifications has been presented. Section 3 demonstrates the proposed design and section 4 given implementation results of proposed design. Further, section 5 concludes the article.

\section{TURBO DECODER DESIGN SPECIFICATIONS}

In SISO decoder, MAP algorithm performs calculations of branch metrics $(\gamma)$, the forward $(\alpha)$, backward $(\beta)$ state metrics and the log likelihood ratio (LLR) values for data block of size $-\mathrm{N}$. Here, back ward state metric computation has to wait till the last input received. Then, LLR calculation depends on branch metrics $(\gamma)$, forward $(\alpha)$, backward $(\beta)$ state metric values. Therefore, this process gives high latency in conventional MAP decoders due to the calculation of forward state metrics and then calculation of backward state metrics in all trellis states to compute LLR values. Therefore, a large memory is necessary to store input data and both forward and backward state metric values, results in larger overhead in the system. So, it is impractical to develop such a decoder with very high latency and large overhead for storage elements. 


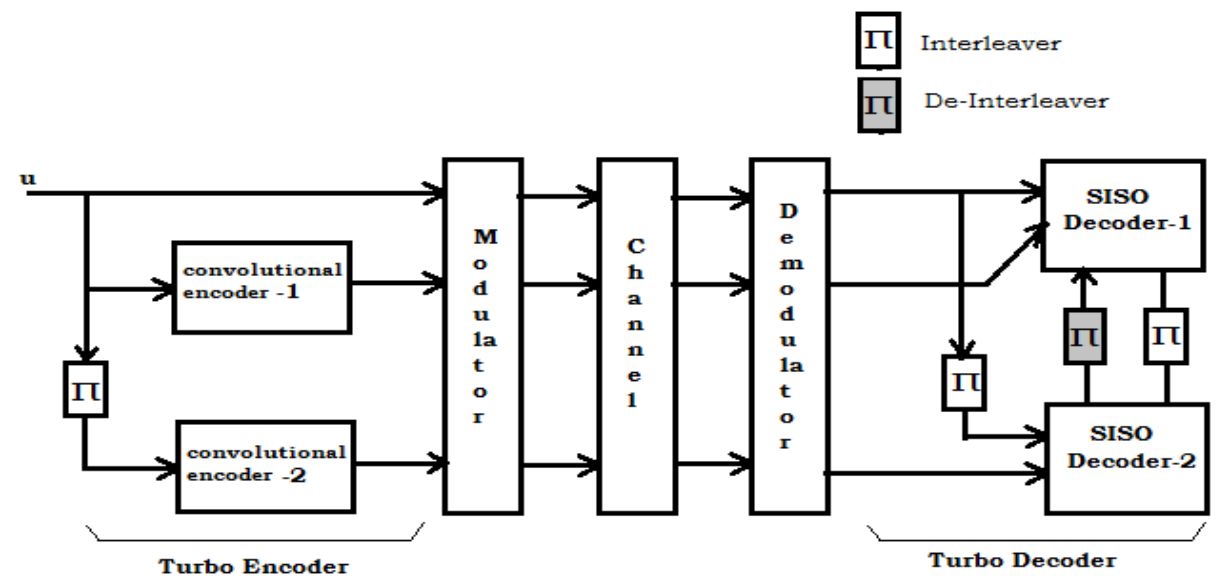

Figure 1 Structure of Turbo Decoder

The vast developments in digital CMOS technologies made possible of implementing complex algorithms on low-cost silicon chips. Channel decoding is an ever interesting and challenging task for VLSI design engineers to implement its optimal system. From design and implementation point of view, it is clear that straightforward computation of equations of original MAP algorithm is very complex and it implies two major problems.

- Large memories requirement for restoring whole transmitted date and intermediate metrics.

- More number of multiplication operations, increases complexity of decoder and it occupies more silicon area.

The first problem is resorting by an approximated version of MAP algorithm known as Sliding window (SW) MAP algorithm [10-12], where global block of data is separated into consecutive sub-blocks or windows to apply equations separately to perform effective parallel decoding at block level.

The second problem has been solved by re-formulating the equations in the MAP algorithm into the logarithmic version. Thus, complex multiplications replaced with additions, as multiplication takes longer execution time and occupies 10 to 40 times more silicon area than a simple addition, thus to reduce circuit complexity and silicon area.

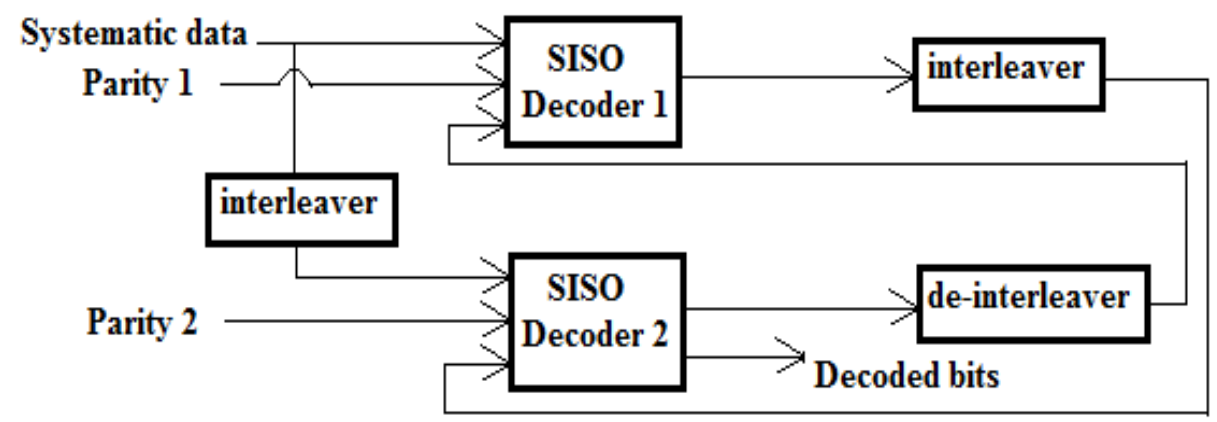

Figure 2 Conventional Turbo Decoder

\subsection{Interleaver Block}

Interleaving process in Turbo decoder is a very critical step in attaining high speed decoding and this process involved in complex computations like computing the remainder of multiplication and division. QPP interleaver is a 3GPP LTE TS specified interleaver for turbo 
decoder of 4G-LTE channel code, therefore finding alternate computing methods to minimize computing resources, latency in accessing memory is an active research in interleaver block.

\subsection{LUT/Memory based Interleaver}

In this approach, an interleaving table with pre-computed addresses for input block size and this table will be accessed during the forthcoming decoding iterations. In a single iteration of decoding, two processes i.e. interleaving and de-interleaving will be carried out. Therefore, this process sense to share pre-stored interleaver table for both interleaving and de-interleaving operation. The draw back in this model is that table occupies more on chip memory. It is not suitable for lengthy block sizes as, a greater number of pre-defined addresses has to be stored in the interleaving table, and thus it uses huge area of the system as given in [22].

\subsection{On-The-Fly Computation based Interleaver:}

As explained in Ref [25], On-the-fly IAG computation approach can be preferred to avoid memory problem presented in the above LUT based Interleaver. It was a simplified recursive QPP interleaver with On-The-Fly IAG computation approach.
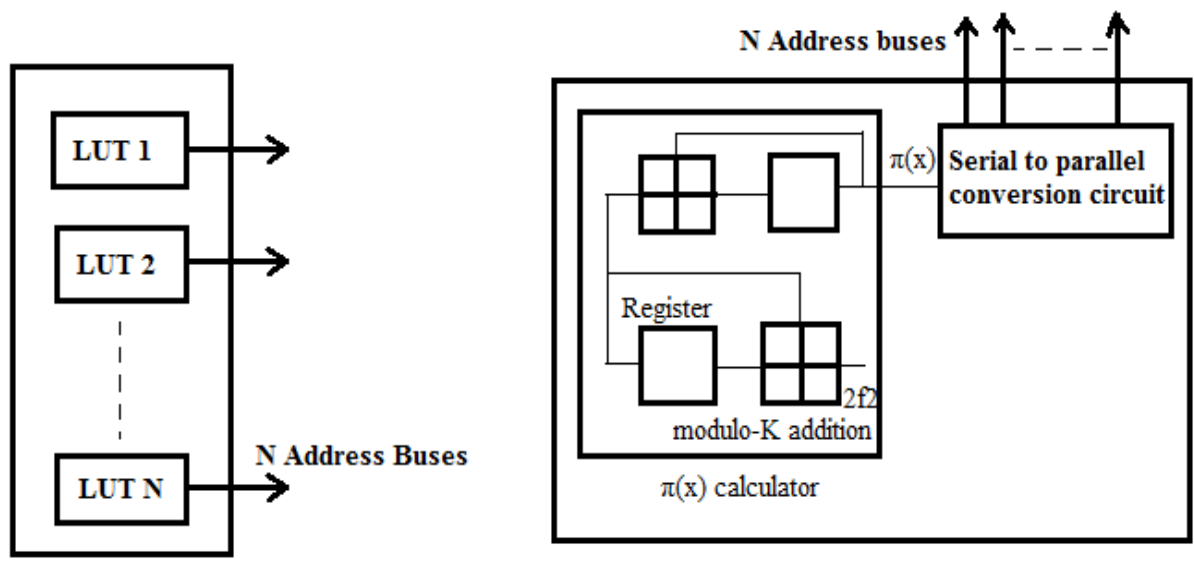

Figure 3 (a) LUT based (b) on-the-Fly Computation based QPP Interleaver

\section{PROPOSED SIMPLIFIED TURBO DECODER}

The efficiency in FPGA architectures will be increased by stream-based signal processing. But, in MAP algorithms direct stream-based processing is not possible as output/input LLRs of BCJR decoder depends o present and past results of that state. Therefore, in proposed architecture, the BCJR decoder process has been organized in three micro pipeline stages as shown in Figure. 4 and in the micro-pipeline stages the two soft-in and soft-out decoders named as SISO-1 of inputs systematic, parity-1, interleaved a-priori data and SISO-2 of inputs interleaved systematic, parity-2, interleaved a-priori data are processed in parallel.

Proposed three stage micro pipelines BCJR decoding:

- In the first micro pipeline stage, the SISO-1 and SISO-2 are processed in parallel with the two inputs and produced extrinsic information output.

- In the second micro-pipeline stage the produced output information is processed to interleaved/ De-interleaved block.

- Finally, in the third micro-pipeline stage the third input of de-interleaved a-priori data to SISO-1 and interleaved a-priori data to SISO-2 decoder blocks is processed. 


\section{Proposed Simplified Computations in BCJR decoder:}

- Calculation of branch metrics is the first step in the proposed architecture for a given length of bits.

- In the second step, computation of forward state metrics and backward state metrics simultaneously.

- Calculation of posteriori LLRs

This process of recursive process will complete into eight numbers of iterations. This is the execution of one turbo decoder; similarly, eight numbers of decoders are performing the same operation in parallel for parallel Turbo decoder architecture.

As decoding process in turbo decoder is a sequential process, the proposed architecture consists of only one decoder of Max log-MAP decoder as shown in Figure 5. In the iterative process, input data for the first decoder, should go through the second decoder and the deinterleaver. Similarly, for the second decoder, the input data should go through the first decoder and the interleaver. In this process, two MAP blocks are not optimal and it will not process more than a half period.

Therefore, in the proposed turbo decoder architecture, one block of Max-Log-MAP decoder exists instead of two as in conventional design. In the proposed architecture, control logic unit generates control signals for multiplexers, interleaver/de-interleaver and MAP decoder in order to process the iterations.

The details of turbo decoder proposed are:

Block size: 40 to 6144

Minimum Number of iterations: 8

Algorithm: Max Log MAP Algorithm

Interleaving: Recursive Computation Approach

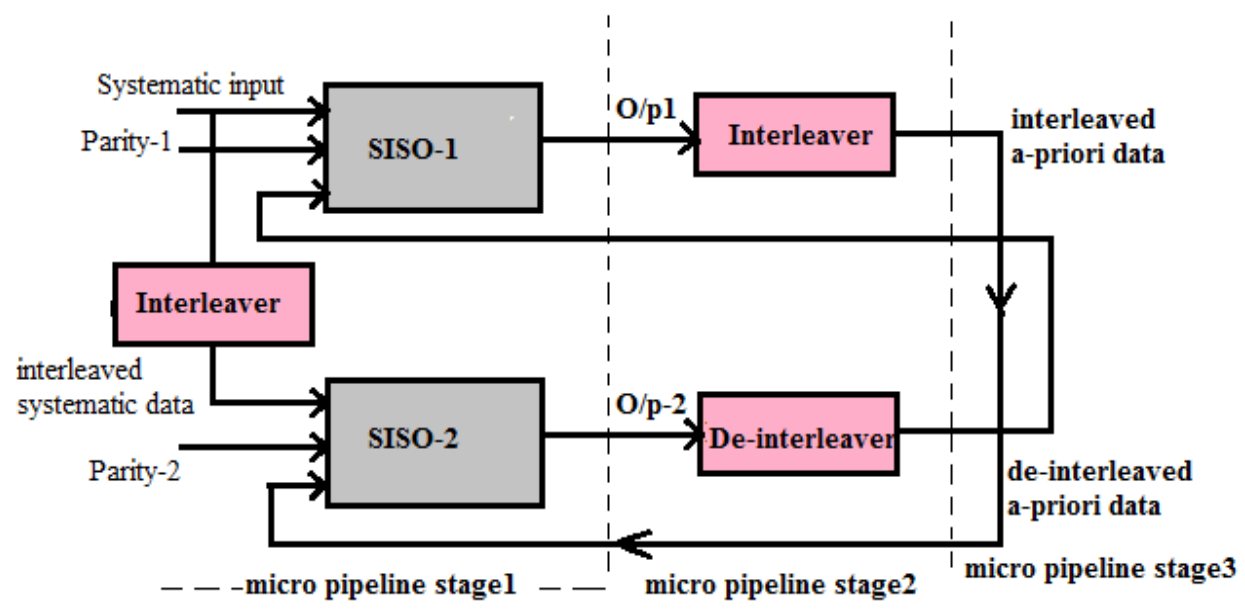

Figure 4 Proposed 3-Stage Pipeline Turbo Decoder

Proper approximations for VLSI implementation is one good solution for reducing circuit complexity, but such approximation is a trade-off between error correction capability and processing delay in metrics updating. The speed of this part of the decoder is extremely important, due to the fact that backward metric computation is a recursive operation, where the set of newly generated metrics is the basis for the following repetition of the updating process. In this kind of loop algorithms, the pipelining is splitting of a complex combinational operation into simpler processing steps is of no help and the only possibility is to reduce as much as possible the delay along the combinational path running from the input to the output metrics. 
In the first step, Branch metrics will be computed with received soft systematic bit $\mathrm{x}(\mathrm{k})$, received soft parity bit y $(\mathrm{k})$ and a-priori information $\lambda_{\text {in }}(\mathrm{k})$,

$$
\begin{gathered}
\gamma_{00}(k)=\mathrm{x}(\mathrm{k})+\lambda_{i n}(\mathrm{k})+\mathrm{y}(\mathrm{k}) \\
\gamma_{01}(k)=\mathrm{x}(\mathrm{k})+\lambda_{\text {in }}(\mathrm{k})-\mathrm{y}(\mathrm{k}) \\
\gamma_{10}(k)=-\mathrm{x}(\mathrm{k})-\lambda_{\text {in }}(\mathrm{k})+\mathrm{y}(\mathrm{k}) \\
\gamma_{11}(k)=-\mathrm{x}(\mathrm{k})+\mathrm{y}(\mathrm{k})-\lambda_{\text {in }}(\mathrm{k})
\end{gathered}
$$

Simplified forward state metrics $(\alpha)$ in the proposed architecture,

$$
\begin{aligned}
& \alpha_{0}=\max \left(\alpha_{0}^{\prime}+\gamma_{00}, \alpha_{1}^{\prime}+\gamma_{11}\right) \\
& \alpha_{1}=\max \left(\alpha_{2}^{\prime}+\gamma_{10}, \alpha_{3}^{\prime}+\gamma_{01}\right) \\
& \alpha_{2}=\max \left(\alpha_{4}^{\prime}+\gamma_{01}, \alpha_{5}^{\prime}+\gamma_{10}\right) \\
& \alpha_{3}=\max \left(\alpha_{6}^{\prime}+\gamma_{11}, \alpha_{7}^{\prime}+\gamma_{00}\right) \\
& \alpha_{4}=\max \left(\alpha_{0}^{\prime}+\gamma_{11}, \alpha_{1}^{\prime}+\gamma_{00}\right) \\
& \alpha_{5}=\max \left(\alpha_{2}^{\prime}+\gamma_{01}, \alpha_{3}^{\prime}+\gamma_{10}\right) \\
& \alpha_{6}=\max \left(\alpha_{4}^{\prime}+\gamma_{10}, \alpha_{5}^{\prime}+\gamma_{01}\right) \\
& \alpha_{7}=\max \left(\alpha_{6}^{\prime}+\gamma_{00}, \alpha_{7}^{\prime}+\gamma_{11}\right)
\end{aligned}
$$

Simplified backward state metrics $(\beta)$ in the proposed architecture,

$$
\begin{aligned}
& \beta_{0}^{\prime}=\max \left(\beta_{0}+\gamma_{00}, \beta_{4}+\gamma_{11}\right) \\
& \beta_{1}^{\prime}=\max \left(\beta_{0}+\gamma_{11}, \beta_{4}+\gamma_{00}\right) \\
& \beta_{2}^{\prime}=\max \left(\beta_{1}+\gamma_{10}, \beta_{5}+\gamma_{01}\right) \\
& \beta_{3}^{\prime}=\max \left(\beta_{1}+\gamma_{01}, \beta_{5}+\gamma_{10}\right) \\
& \beta_{4}^{\prime}=\max \left(\beta_{2}+\gamma_{01}, \beta_{6}+\gamma_{10}\right) \\
& \beta_{5}^{\prime}=\max \left(\beta_{2}+\gamma_{10}, \beta_{6}+\gamma_{01}\right) \\
& \beta_{6}^{\prime}=\max \left(\beta_{3}+\gamma_{11}, \beta_{7}+\gamma_{00}\right) \\
& \beta_{7}^{\prime}=\max \left(\beta_{3}+\gamma_{00}, \beta_{7}+\gamma_{11}\right)
\end{aligned}
$$

Soft output L can be computed as,

$$
\begin{gathered}
L=\max \left(\alpha_{0}^{\prime}+\beta_{0}+\gamma_{00}, \alpha_{1}^{\prime}+\beta_{4}+\gamma_{00},\right. \\
\alpha_{2}^{\prime}+\beta_{5}+\gamma_{01}, \alpha_{3}^{\prime}+\beta_{1}+\gamma_{01}, \\
\alpha_{4}^{\prime}+\beta_{2}+\gamma_{01}, \alpha_{5}^{\prime}+\beta_{6}+\gamma_{01}, \\
\left.\alpha_{6}^{\prime}+\beta_{7}+\gamma_{00}, \alpha_{7}^{\prime}+\beta_{3}+\gamma_{00}\right)- \\
\operatorname{Max}\left(\alpha_{0}^{\prime}+\beta_{4}+\gamma_{11}, \alpha_{1}^{\prime}+\beta_{0}+\gamma_{11},\right. \\
\alpha_{2}^{\prime}+\beta_{1}+\gamma_{10}, \alpha_{3}^{\prime}+\beta_{5}+\gamma_{10} \\
\alpha_{4}^{\prime}+\beta_{6}+\gamma_{10}, \alpha_{5}^{\prime}+\beta_{2}+\gamma_{10} \\
\left.\alpha_{6}^{\prime}+\beta_{3}+\gamma_{11}, \alpha_{7}^{\prime}+\beta_{7}+\gamma_{11}\right)
\end{gathered}
$$

Simplified soft output with common $\gamma_{u v}$, is given as

$L=\max \left(\max \left(s_{0}, s_{1}\right)+\gamma_{00}, \max \left(s_{2}, s_{3}\right)+\gamma_{01}-\max \left(t_{0}, t_{1}\right)+\gamma_{11}, \max \left(t_{2}, t_{3}\right)+\gamma_{10}\right)$

Where,

$$
\begin{aligned}
& s_{0}=\max \left(\alpha_{0}^{\prime}+\beta_{0}, \alpha_{1}^{\prime}+\beta_{4}\right) \\
& s_{1}=\max \left(\alpha_{6}^{\prime}+\beta_{7}, \alpha_{7}^{\prime}+\beta_{3}\right) \\
& s_{2}=\max \left(\alpha_{2}^{\prime}+\beta_{5}, \alpha_{3}^{\prime}+\beta_{1}\right) \\
& s_{3}=\max \left(\alpha_{4}^{\prime}+\beta_{2}, \alpha_{5}^{\prime}+\beta_{6}\right) \\
& t_{0}=\max \left(\alpha_{0}^{\prime}+\beta_{4}, \alpha_{1}^{\prime}+\beta_{0}\right)
\end{aligned}
$$




$$
\begin{aligned}
& t_{1}=\max \left(\alpha_{6}^{\prime}+\beta_{3}, \alpha_{7}^{\prime}+\beta_{7}\right) \\
& t_{2}=\max \left(\alpha_{2}^{\prime}+\beta_{1}, \alpha_{3}^{\prime}+\beta_{5}\right) \\
& t_{3}=\max \left(\alpha_{4}^{\prime}+\beta_{6}, \alpha_{5}^{\prime}+\beta_{2}\right)
\end{aligned}
$$

The extrinsic information/ a-posteriori information $\lambda_{\text {out }}(\mathrm{k})$ can be calculated with the aid of $\mathrm{L}(\mathrm{k}), \mathrm{x}(\mathrm{k})$ and $\mathrm{y}(\mathrm{k})$ as,

$$
\lambda_{\text {out }}(k)=\frac{1}{2} L(k)-x(k)-\lambda_{\text {in }}(\mathrm{k})
$$

Where, $\mathrm{x}(\mathrm{k})$ is the received soft systematic information, $\lambda_{\text {in }}(\mathrm{k})$ is the a-priori information.

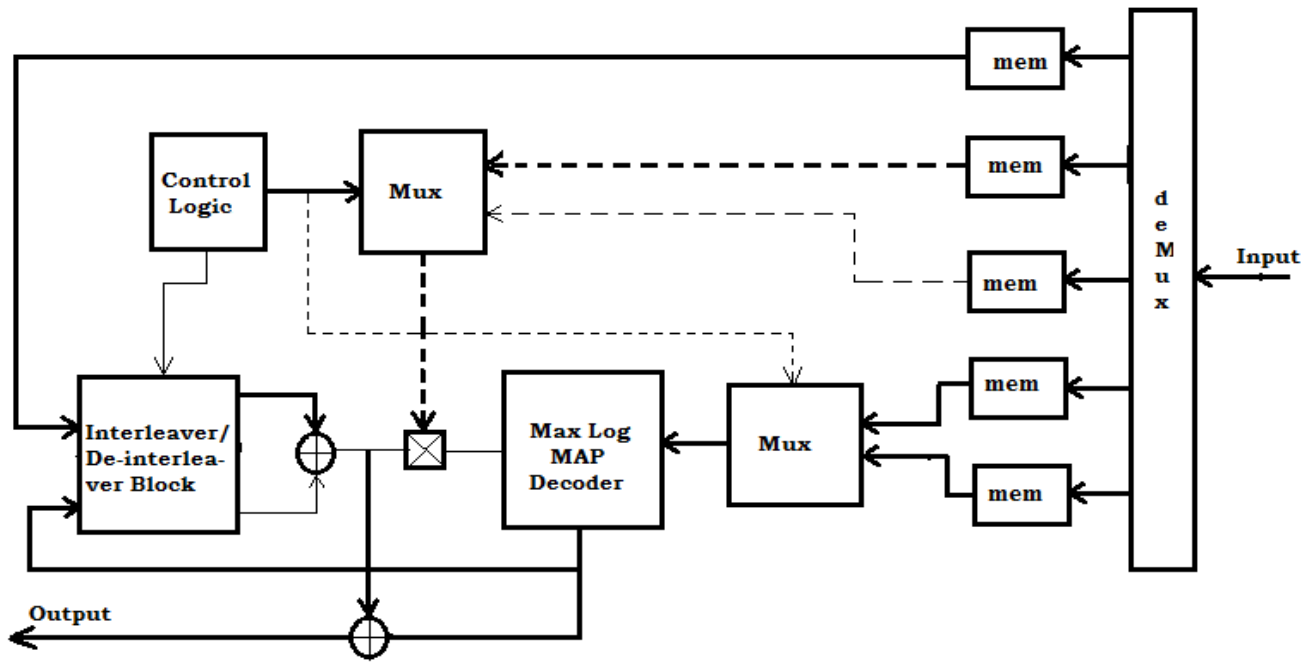

Figure 5 Data routing in the proposed Turbo decoder

The Control logic unit sends the signal to multiplexers, interleaver /de-interleaver to start iterative process of Max-Log MAP Decoder. Once loading of input data has been done in stage 1 , then computation of branch metrics gets started, then the parallel process of $\alpha$ and $\beta$ which in turn to compute LLRs of that half iteration. This process continues till the maximum value is achieved/ one complete Max-log-MAP decoding result is produced. Figure. 5 shows processing of loading and selecting the input data for BCJE decoder in terms of data routing.

\section{RESULTS AND DISCUSSION}

The proposed optimized Turbo Decoder has been designed using Max-log-MAP algorithm. The design developed with Verilog HDL and synthesized and implemented on Zynqzed FPGA board. The proposed simplifications in BCJR decoder and the results of hardware resource utilization for respective techniques applied has been listed in Table1 Figure.6 (a) is RTL schematic of proposed simplified design, which has single BCJR decoder with Interleaver block and control unit as designed in our proposed architecture. Also, Figure.6 (b) shows, system generated RTL schematic of proposed architecture of simplified state metric computation blocks has been given. These simplified computations reduce memory requirement and computational latency as well. 


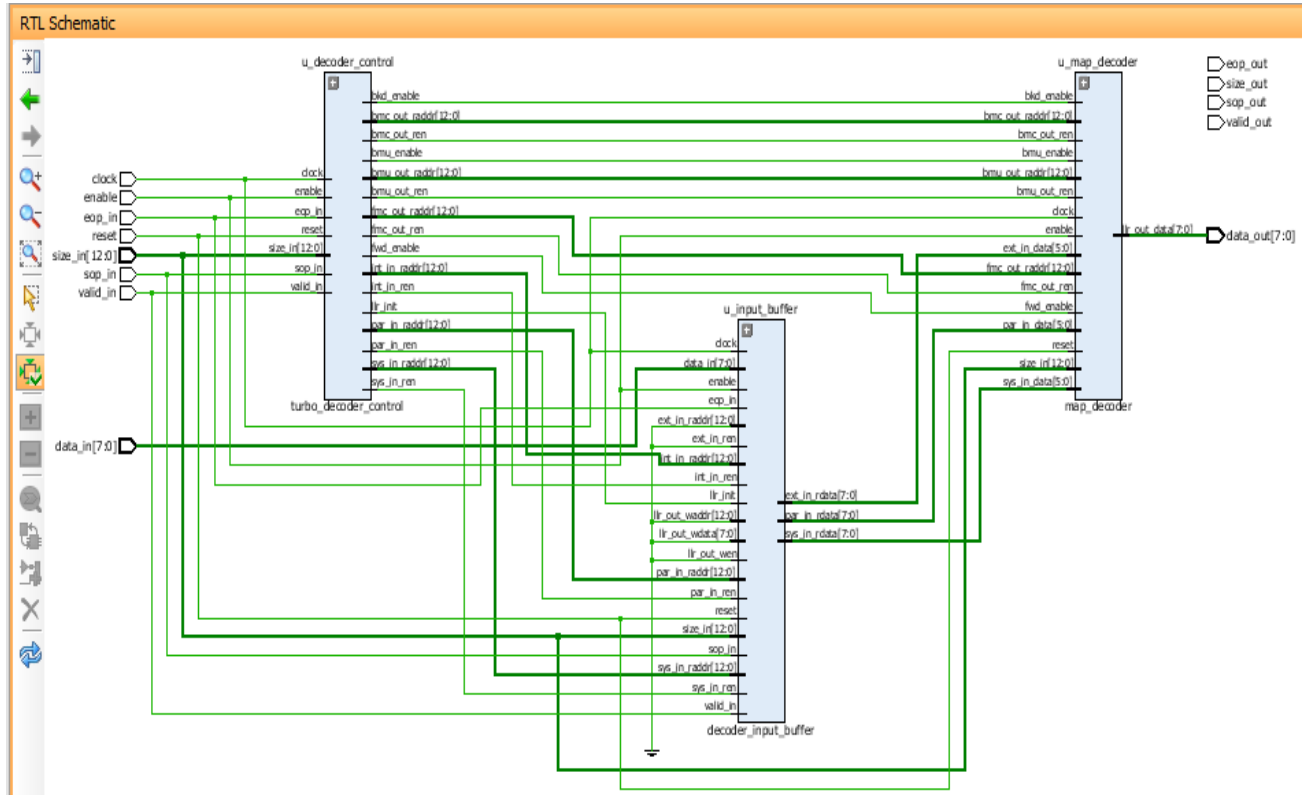

Figure 6 RTL Schematic of Proposed Turbo decoder design

Figure.6 is RTL schematic of proposed simplified design, which has single BCJR decoder with Interleaver block and control unit as designed in our proposed architecture. In Figure.7, system generated RTL schematic of our proposed architecture of simplified state metric computation blocks has been given. These simplified computations reduces memory requirement and computational as well.

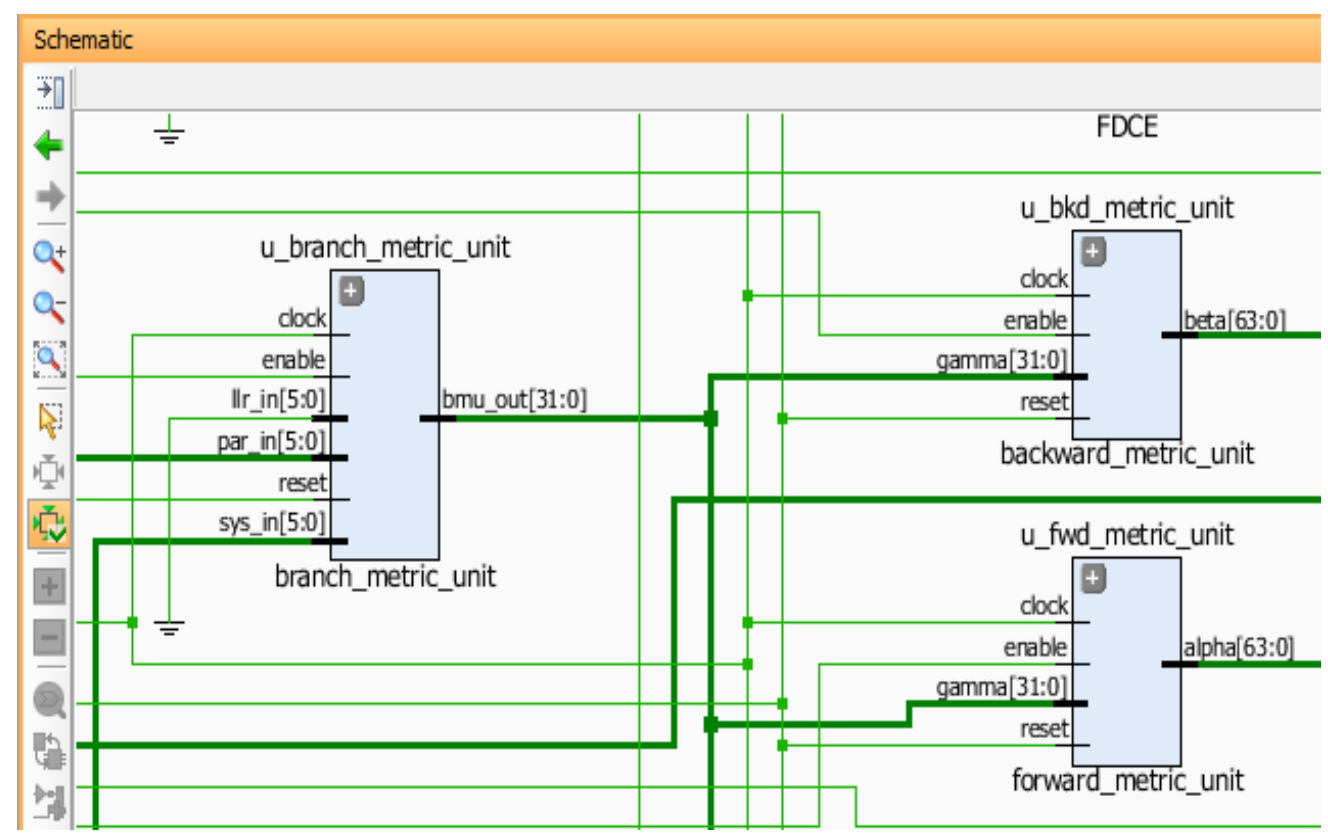

Figure 7 RTL Schematic of Computation Units in MAP Decoder

From Table.1, it is observed that, the optimization techniques like performance retiming and area optimization results in less number of LUTs, Flipflops and BRAM. The same design without applying optimization occupied more number of hardware memories as shown in first column of the Table.1. 
Table 1 Hardware Resource Utilization for various techniques applied to the proposed Design

\begin{tabular}{|c|c|c|c|c|c|}
\hline Resource & $\begin{array}{c}\text { Utilization \& } \\
\text { (Utilization \%) } \\
\text { (post synthesis) } \\
\text { without } \\
\text { Optimization }\end{array}$ & $\begin{array}{c}\text { Utilization } \\
\text { (Utilization \%) } \\
\text { (post synthesis) } \\
\text { With optimization } \\
\text { and Without } \\
\text { resource Sharing }\end{array}$ & $\begin{array}{c}\text { Utilization } \\
\text { (Utilization \%) } \\
\text { (post synthesis) } \\
\text { With resource } \\
\text { sharing }\end{array}$ & $\begin{array}{c}\text { Utilization } \\
\text { (Utilization \%) } \\
\text { ( post } \\
\text { implementation) } \\
\text { With performance } \\
\text { retiming and area } \\
\text { optimization }\end{array}$ & Available \\
\hline FF & $1150(1.08 \%)$ & $840(0.79 \%)$ & $809(0.76 \%)$ & $809(0.76 \%)$ & 106400 \\
\hline LUT & $1497(2.81 \%)$ & $1130(2.12 \%)$ & $1081(2.03 \%)$ & $1068(2.01 \%)$ & 53200 \\
\hline I/O & $29(14.5 \%)$ & $38(19 \%)$ & $38(19 \%)$ & $34(17 \%)$ & 200 \\
\hline BRAM & $46(32.86 \%)$ & $32(22.86 \%)$ & $32(22.86 \%)$ & $32(22.86 \%)$ & 140 \\
\hline BUFG & $1(3.12 \%)$ & $1(3.12 \%)$ & $1(3.12 \%)$ & $1(3.12 \%)$ & 32 \\
\hline
\end{tabular}

\section{CONCLUSION}

In this article, proposed design and implementation of Turbo decoder as been described. The main purpose of this design is to minimize the hardware resource utilization like number of LUTs and memory registers by applying VLSI optimization techniques as explained in section 3. Also, for further improvement in the design, sliding window method can be applied at Maxlog MAP decoder block to improve throughput and minimize still more resource utilization.

Applying deep learning algorithm for parallel processing of BCJR decoders further research can be done and it is a challenging task to a design engineer and such type of application of deep learning algorithms in this work can be still reduced the latency and hardware requirement.

\section{ACKNOWLEDGEMNT}

This research received research grant from Department of Science and Technology (DST), Government of India. The Authors would like to thank Women Scientist Scheme (WOS-A), Department of Science and Technology (DST), Government of India for providing financial support to carry out the project work under KIRAN DIVISION, Grant No. SR/WOS-A/ET-72, at Research Centre, Department of ECE, Sree Vidyanikethan Engineering College, Tirupati, Andhra Pradesh, India.

\section{REFERENCES}

[1] IEEE Standards for Local and Metropolitan Area Networks Part 16: Air Interface for Fixed and Mobile Broadband Wireless Access Systems, Inst. Electrical and Electronics Engineers (IEEE) Std. IEEE 802.16m-2009, 2009.

"Worldwide Interoperability for http://www.wimaxforum.org/index.htm.

Microwave Access

(WiMAX),"

[3] Technical Specification Group Radio Access Network; Evolved Universal Terrestrial Radio Access (E-UTRA) (2012); Multiplexing and channel coding, 3rd Generation Partnership Project Std. TS 36.212 v11.0.0, 2012.

[4] L. Christodoulou, O. Abdul-Hameed, A. M. Kondoz and J. Calic, Adaptive Sub frame Allocation for Next Generation Multimedia Delivery Over Hybrid LTE Unicast Broadcast, IEEE Trans. Broadcast., 62(3), 2016, 540-551. 
[5] L. Bahl, J. Cocke, F. Jelinek and J. Raviv, Optimal decoding of linear codes for minimizing symbol error rate, IEEE Trans. Info. Theory, 20(2), 1974, 284-287.

[6] Robertson P, Villebrun E, Hoeher P, A Comparison of Optimal and Sub-Optimal MAP decoding Algorithms Operating in the Log-Domain, Proc. International Conference on Communications (ICC '95), Seattle, Washington, USA, 1995, 1009-1013.

[7] Talakoub, L.Sabeti, B. Shahrrava and M. Ahmadi, An improved Max-Log-Map algorithm for turbo decoding and turbo equalization, IEEE Trans. Ins. Meas., 56, 2007, 1058-1063.

[8] Papaharalabos S, Mathiopoulos PT, Masera G, Martina M, on optimal and near-optimal turbo decoding using generalized max operator, IEEE Communication Letters, 13(7), 2009, 522-524.

[9] L. Christodoulou, O. Abdul-Hameed, A. M. Kondoz and J. Calic, Adaptive Subframe Allocation for Next Generation Multimedia Delivery Over Hybrid LTE Unicast Broadcast, IEEE Trans. Broadcast., 62(3), 2016, 540-551.

[10] Simplified Log-MAP algorithm for very low complexity turbo decoder hardware architectures. IEEE Trans. Ins. Meas., 63(3), 2013, 531-537.

[11] A. J. Viterbi, An intuitive justification and a simplified implementation of the MAP decoder for convolutional codes, IEEE J. Sel. Areas Commun., 16(2), 1998, 260-264.

[12] S. A. Barbulescu, Iterative decoding of turbo codes and other concatenated codes, Ph.D. dissertation, University of South Australia, 1996.

[13] S. S. Pietrobon, Efficient implementation of continuous MAP decoders and lexity a synchronization technique for turbo decoders, in Proc. Int. Symp. Inform.Theory Appl., Victoria, B.C., Canada, 1996, 586-589.

[14] R. Shrestha and R. Paily, Hardware implementation of Max-Log MAP algorithm based on Mac Laurin series for turbo decoder, Proceedings on International Conference in Communications and Signal Processing (ICCSP), 2011, 509-511.

[15] M. Martina and G. Masera, State Metric Compression Techniques for Turbo Decoder Architectures, IEEE Transactions on Circuits and Systems, 58(5), 2011, 1119-1128.

[16] M.U. Karim, M.U.K. Khan and Y.M. Khawaja, An area reduced, speed optimized implementation of Viterbi decoder, International Conference on Computer Networks and Information Technology (ICCNIT), 2011, 93-98.

[17] C.H. Lin, C.Y. Chen and A.Y. Wu, Area-Efficient Scalable MAP Processor Design for HighThroughput Multi-standard Convolutional Turbo Decoding, IEEE Transactions on very large scale integration VLSI systems, 19(2), 2011, 305-318.

[18] Yang Sun and Joseph R.Cavallaro, Efficient hardware implementation of a highly-parallel 3GPPLTE/LTE-advance turbo decoder, Integration, the VLSI Journal, 44, 2011, 305-315.

[19] C. Condo, M. Martina, and G. Masera, VLSI implementation of multi-mode turbo/LDPC decoder architecture, IEEE Transactions on Circuits and Systems I, 60(6), 2013, 1441-1454.

[20] R. Shrestha and R. Paily, High-Throughput Turbo Decoder With Parallel Architecture for LTE Wireless Communication Standards, IEEE Transactions On Circuits And Systems I: Regular Papers, 2014, 2699-2710. 
[21] A. Li, L. Xiang, T. Chen, R. G. Maunder, B. M. Al-Hashimi and L. Hanzo, VLSI Implementation of Fully Parallel LTE Turbo Decoders, IEEE Access, 4, 2016, 323-346.

[22] E. Sujatha, C. Subhas and M. N. Giri Prasad, High performance turbo encoder using mealy FSM state encoding technique, International Journal of Engineering and Technology (UAE), 7(2.33), 2018, 255-258.

[23] E. Sujatha, C. Subhas and M. N. Giri Prasad, Design aspects of MAP Turbo decoder for wireless communication Systems, International Journal of Engineering Research in Electronics and Communication Engineering, 3(3), 2017, 1-8.

[24] E. Sujatha, C. Subhas and M. N. Giri Prasad, Performance improvement of Turbo decoder using VLSI optimization Techniques, IEEE International Conference on Vision, Towards Emerging Trends in Communication and Networking (ViTECoN), 2019.

[25] E. Sujatha, C. Subhas and M. N. Giri Prasad, Simplified on-the-fly IAG QPP interleaver for turbo channel coding scheme, International Journal of Recent Technology and Engineering, 8(3), 2019, 1443-1448. 